\title{
Usefulness and problems of cytological examination by endobronchial ultrasound-guided transbronchial needle aspiration in the diagnosis of mediastinal lymphadenopathy: A retrospective single-centre study
}

\author{
KAORI SANDOH, MITSUAKI ISHIDA, KIMIAKI OKANO, HIROKO ITO and KOJI TSUTA \\ Department of Pathology and Clinical Laboratory, Kansai Medical University, Hirakata, Osaka 573-1010, Japan
}

Received December 10, 2020; Accepted April 29, 2021

DOI: $10.3892 / \mathrm{mco} .2021 .2300$

\begin{abstract}
Endobronchial ultrasound-guided transbronchial needle aspiration (EBUS-TBNA) is a minimally invasive and useful technique for sampling mediastinal lymph nodes. High sensitivity and specificity of EBUS-TBNA in staging lung cancer have been reported. However, few studies have addressed the results of EBUS-TBNA in the diagnosis of mediastinal lymphadenopathy with or without lung cancer, since various neoplastic and non-neoplastic conditions can involve the mediastinal lymph nodes. The present study analysed the results of the cytological examination of mediastinal lymphadenopathy using EBUS-TBNA in Kansai Medical University Hospital (Hirakata, Japan). A total of 41 consecutive patients with mediastinal lymphadenopathy who underwent EBUS-TBNA between January 2008 and December 2019 in Kansai Medical University Hospital were enrolled. This cohort included 29 males and 12 females. Cytological analyses demonstrated that $16 / 17$ (94.1\%) patients with metastatic carcinoma were diagnosed as having carcinoma (malignancy). The sensitivity, specificity and positive predictive value of the cytological examination in cases of carcinoma were 94.1, 100 and 100, respectively. However, only 3/11 (27.3\%) patients with sarcoidosis were diagnosed by cytological examination. The present study demonstrated that EBUS-TBNA may be a useful method for detecting metastatic carcinoma in the mediastinal lymph nodes. However, the detection rate of epithelioid granuloma, which is a characteristic feature of sarcoidosis, was low in the present cohort. Hence, an improvement in the sampling technique is necessary.
\end{abstract}

Correspondence to: Dr Mitsuaki Ishida, Department of Pathology and Clinical Laboratory, Kansai Medical University, 2-5-1 Shinmachi, Hirakata, Osaka 573-1010, Japan

E-mail: ishidamt@hirakata.kmu.ac.jp

Key words: endobronchial ultrasound-guided transbronchial needle aspiration, mediastinal lymph node, lymphadenopathy, cytology, lung cancer, sarcoidosis

\section{Introduction}

Various neoplastic and non-neoplastic conditions can involve the mediastinal lymph nodes; hence, correct histopathological diagnosis is very important for appropriate treatment of patients with mediastinal lymphadenopathy. Endobronchial ultrasound-guided transbronchial needle aspiration (EBUS-TBNA) is a technique in which the needle is advanced through the channel of the bronchoscope with real-time visualisation of the target lesion by ultrasound and obtaining a sample by fine-needle aspiration from the target lesion (1). EBUS-TBNA has been recognised as a useful and minimally invasive technique for sampling mediastinal lymph nodes $(1,2)$. The high sensitivity and specificity of EBUS-TBNA in staging for non-small cell lung carcinoma (irrespective of the presence of metastasis in the mediastinal lymph nodes) has been well recognised (1). Therefore, the current guidelines recommend EBUS-TBNA as the first-line approach for lymphadenopathy of the mediastinal lymph nodes in patients with lung cancer (3).

It is also very important to determine the cause of lymphadenopathy of the mediastinal lymph nodes in patients without lung cancer, i.e., reactive/inflammatory change (including sarcoidosis) or neoplastic change (such as malignant lymphoma). The usefulness of EBUS-TBNA in the diagnosis of lymphadenopathy in patients without lung cancer has also been reported (4). However, few studies have evaluated the diagnostic results of mediastinal lymphadenopathy using EBUS-TBNA in consecutive patients with or without lung cancer $(4,5)$. The aim of the present study was to analyse the results of the cytological examination using EBUS-TBNA in the diagnosis of mediastinal lymphadenopathy in a single centre, and also discuss the usefulness and diagnostic problems in cytological examination using EBUS-TBNA in patients with mediastinal lymphadenopathy.

\section{Patients and methods}

Patient selection. We enrolled 41 consecutive patients who underwent EBUS-TBNA for the cytological and histopathological diagnosis of mediastinal lymphadenopathy from January 2008 to December 2019 at the Kansai Medical University Hospital. 
This study was conducted in accordance with the Declaration of Helsinki, and the study protocol was approved by the institutional review board of the Kansai Medical University Hospital (protocol no. 160954).

Cytological analyses. The cytological specimens obtained by EBUS-TBNA were fixed in alcohol and subsequently routinely stained with Papanicolaou stain; air-dried cytological specimens were also stained with Giemsa stain according to routine hospital procedures. No liquid-based cytology was performed in the present study. Furthermore, rapid on-site evaluation (ROSE) of the cytological specimens was not performed.

The cytological characteristics were independently evaluated by one diagnostic pathologist and two cytopathologists. Disagreements were resolved by reassessment using a multi-headed microscope.

Histological analyses. Two diagnostic pathologists independently evaluated the histopathological features of the biopsy specimens obtained by EBUS-TBNA.

\section{Results}

Clinical features. This cohort included 41 patients (29 males and 12 females), who were aged 22-83 years. The reasons for performing the EBUS-TBNA were as follows: Mediastinal lymphadenopathy of unknown cause (16 patients), suspicion of sarcoidosis (15 patients), lung cancer (9 patients), and lymphadenopathy due to metastatic tumour (1 patient). There were no severe complications associated with the EBUS-TBNA procedure in the present cohort. The information about node stations sampled was not available for this cohort.

Cytologicalfeatures. Table I summarises the results of the cytological examinations of the samples obtained by EBUS-TBNA in this cohort. Malignancy was seen in 16 patients $(39.0 \%)$, 1 patient $(2.4 \%)$ had suspected malignancy, 22 patients $(53.6 \%)$ had no malignancy, and the sample was inadequate for diagnosis in 2 patients (4.9\%). Patients with malignancy included small cell carcinoma (4 patients), adenocarcinoma (3 patients), squamous cell carcinoma (2 patients), and non-small cell carcinoma ( 7 patients) (Fig. 1). In 3 patients who had no malignancy, only a few epithelioid granulomas were observed; thus, sarcoidosis was suspected.

Histological features and correlation with cytological diagnosis. Table II summarises the results of the histological examination. Carcinoma was diagnosed in 17 patients (adenocarcinoma in 6 patients, small cell carcinoma in 4 patients, squamous cell carcinoma in 3 patients, and non-small cell carcinoma in 4 patients). One patient who had adenocarcinoma was considered cytologically negative because the carcinoma was not visible in the cytological specimens. Thus, 16 of 17 (94.1\%) patients with carcinoma were diagnosed as having carcinoma (positive/malignancy) by cytological examination. Moreover, one patient with suspected malignancy on cytological examination did not have carcinoma on histological examination. The sensitivity, specificity, and positive predictive value of the cytological examination of carcinoma (malignancy) were 94, 100 and 100\%, respectively.
Table I. Summary of the results of the cytological examination of the mediastinal lymph nodes in 41 patients.

\begin{tabular}{lr} 
Results & Patients, $\mathrm{n}(\%)$ \\
\hline Positive/malignancy & $16(39.0)$ \\
Small cell carcinoma & $4(9.8)$ \\
Adenocarcinoma & $3(7.3)$ \\
Squamous cell carcinoma & $2(4.9)$ \\
Non-small cell carcinoma & $7(17.1)$ \\
Suspicious of malignancy & $1(2.4)$ \\
Negative & $22(53.6)$ \\
Insufficient & $2(4.9)$
\end{tabular}

Table II. Summary of the results of the histological examination of the mediastinal lymph nodes in 41 patients.

\begin{tabular}{lr}
\hline Results & Patients $(\%)$ \\
\hline Carcinomas & $17(41.5)$ \\
Small cell carcinoma & $4(9.8)$ \\
Adenocarcinoma & $6(14.6)$ \\
Squamous cell carcinoma & $3(7.3)$ \\
Non-small cell carcinoma & $4 \quad(9.8)$ \\
Benign conditions & $24(58.5)$ \\
Sarcoidosis & $11(26.8)$ \\
Cystic lesion & $2(4.9)$ \\
Inflammatory conditions & $1(2.4)$ \\
Schwannoma & $1(2.4)$ \\
Not specified & $9(22.0)$ \\
\hline
\end{tabular}

The histological diagnosis of the remaining 24 patients revealed benign conditions, which included sarcoidosis (11 patients), benign cystic lesion (2 patients), non-specific inflammatory condition (1 patient), schwannoma (1 patient), and unspecified condition ( 9 patients). Only 3 of 11 patients with sarcoidosis were diagnosed by cytological examination $(27.3 \%)$.

\section{Discussion}

The present study analysed the usefulness and problems associated with EBUS-TBNA-based cytological examination of the mediastinal lymph nodes in our institute. In this cohort, the sensitivity, specificity, and positive predictive value of the cytological examination for carcinoma (malignancy) were 94.1, 100 and 100\%, respectively. However, the diagnostic accuracy for sarcoidosis was low; only 3 of $11(27.3 \%)$ patients with sarcoidosis were cytologically diagnosed as sarcoidosis.

The usefulness of EBUS-TBNA is well known, particularly its high sensitivity and specificity in detecting metastasis in the mediastinal lymph nodes in patients with lung cancer (1). Most of the previously published studies demonstrated that the sensitivity was more than $80 \%$ and the 
A

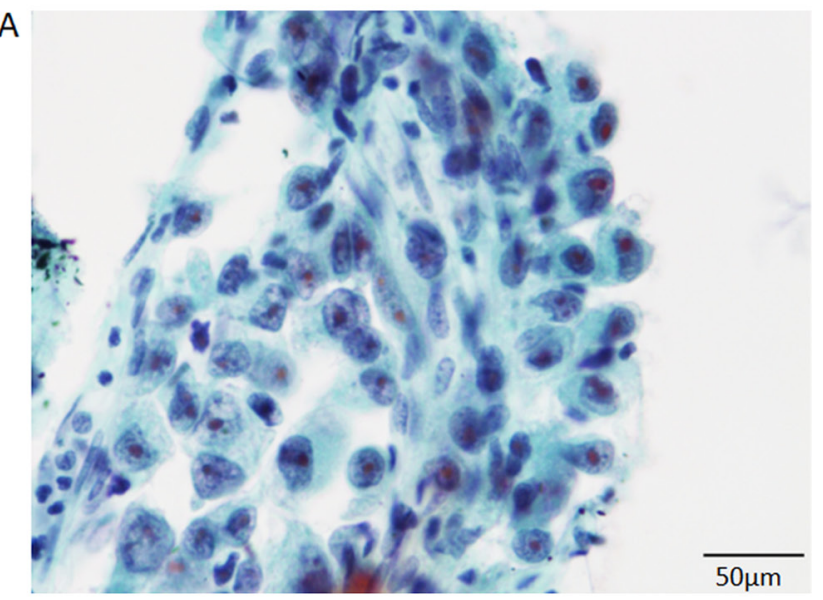

B

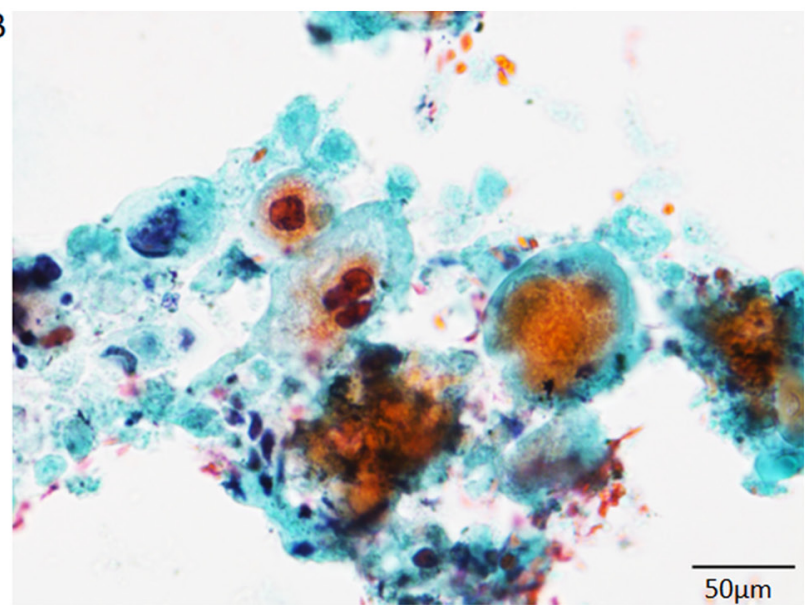

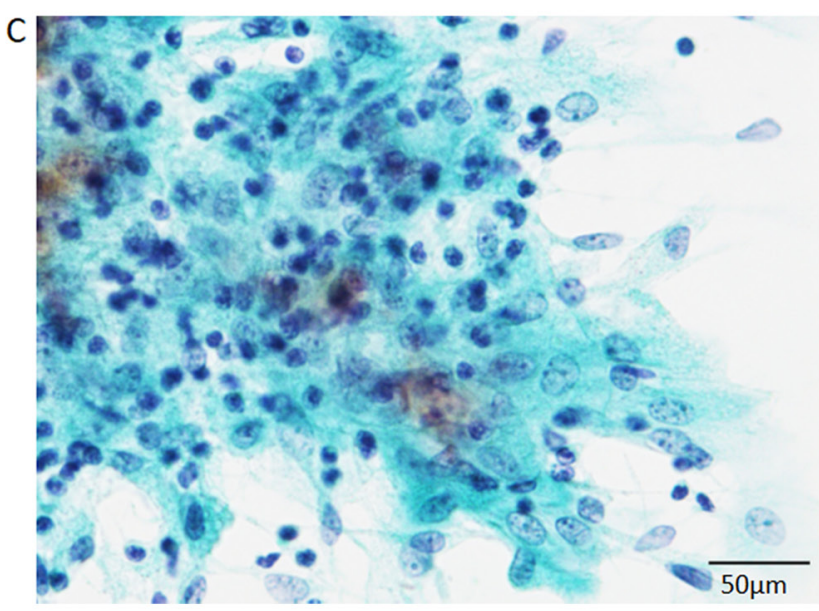

Figure 1. Cytological features of the specimens obtained by endobronchial ultrasound-guided transbronchial needle aspiration. (A) Adenocarcinoma of the lung. The neoplastic cells had large round to oval nuclei containing conspicuous nucleoli and relatively rich cytoplasm (Papanicolaou stain; magnification, x400; scale bar, $50 \mu \mathrm{m}$ ). (B) Squamous cell carcinoma of lung origin. The neoplastic cells had a rich cytoplasm and large nuclei in a necrotic background (Papanicolaou stain; magnification, $\mathrm{x} 400$; scale bar, $50 \mu \mathrm{m}$ ). (C) Sarcoidosis. Epithelioid granuloma was observed in a background of lymphocytes (Papanicolaou stain; magnification, $\mathrm{x} 400$; scale bar, $50 \mu \mathrm{m}$ ).

specificity was almost $100 \%$ (1). Therefore, EBUS-TBNA has been considered as a superior performance tool compared to other staging modalities, such as computed tomography (1). In one series, the sensitivity of diagnosis of mediastinal lymph node metastases from lung cancer was $\sim 70 \%$, and the positive predictive value was $\sim 95 \%$. Moreover, the superiority of liquid-based cytology compared to that of conventional cytological examination has been reported (4). In the present study, sensitivity, specificity, and positive predictive value were $\sim 100 \%$. Thus, EBUS-TBNA is an accurate and useful method for detecting metastatic carcinoma in the mediastinal lymph node in patients with lung cancer.

However, the cytological diagnosis of the mediastinal lymph nodes using EBUS-TBNA might be challenging because the mediastinal lymph nodes can have diverse non-neoplastic conditions, including sarcoidosis. Sarcoidosis is a systemic autoimmune disease characterised by the presence of granulomatous inflammation, which mainly affects the lungs, lymph nodes, eyes, and skin; hilar and mediastinal lymph nodes are also frequently involved (6). The utility of EBUS-TBNA in the diagnosis of sarcoidosis (detection of granulomas) has also been reported $(7,8)$. According to a study of cytological specimens using EBUS-TBNA, well-formed non-necrotising granulomas were noted in $90 \%$ of the patients with sarcoidosis (38/42 patients), and the remaining 4 patients had poorly formed granulomas. The extent of granulomas was as follows: Rare, occasional, and numerous in 26,36 and $38 \%$ of the patients, respectively (9). High sensitivity (>85\%) of EBUS-TBNA in patients with a high clinical suspicion of sarcoidosis has also been reported (10). However, the rate of detection of sarcoidosis (epithelioid granulomas) was low (27.3\%) in the present cohort. This might be due to the amount of the sample because the retrospective analysis of the cytological specimens failed to detect epithelioid granulomas in these cases with epithelioid granulomas. Improvement in the sampling technique and/or method might be required for these patients. The presence of the cytologist at the time of obtaining the cytological specimens and performance of ROSE improved the detection of the targeted lesions in various organs, according to previous studies $(11,12)$. It has been reported that yields of the conventional TBNA with ROSE and EBUS-TBNA with or without ROSE were higher than those of the conventional TBNA without ROSE, although the rates of granuloma detection were not significantly different (7). Moreover, the utility of granuloma detection using ROSE was also reported (9). 
Therefore, ROSE might improve the detection rate of granulomas in EBUS-TBNA.

In conclusion, the present study analysed the usefulness and problems associated with EBUS-TBNA in the cytological examination of the mediastinal lymph nodes in our institute. The sensitivity, specificity, and positive predictive value of the cytological examination for carcinoma (malignancy) were high. Thus, EBUS-TBNA is a useful method for detecting mediastinal lymph node metastasis in patients with lung cancer. However, the diagnostic accuracy of sarcoidosis was low; therefore, an improvement in the sampling technique might be required.

\section{Acknowledgements}

Not applicable.

\section{Funding}

No funding was received.

\section{Availability of data and materials}

All data generated or analyzed during this study are included in this published article.

\section{Authors' contributions}

MI contributed to conception and design of the study. KS, $\mathrm{MI}, \mathrm{KO}$ and $\mathrm{HI}$ contributed to the analyses of the cytological specimens. MI and KT contributed to the analyses of the histological specimens. KS and MI confirm the authenticity of all the raw data, and contributed to drafting of the manuscript, tables and figures. All authors read and approved the final manuscript.

\section{Ethics approval and consent to participate}

The study was conducted in accordance with the Declaration of Helsinki, and the study protocol was approved by the institutional review board of the Kansai Medical University Hospital (Hirakata, Japan; protocol no. 160954). Opt-out consent was obtained from participants of this study due to the retrospective design of the study and no risk to the participants.

\section{Patient consent for publication}

Not applicable.

\section{Competing interests}

The authors declare that they have no competing interests.

\section{References}

1. VanderLaan PA, Wang HH, Majid A and Folch E: Endobronchial ultrasound-guided transbronchial needle aspiration (EBUS-TBNA): An overview and update for the cytopathologist. Cancer Cytopathol 122: 561-576, 2014.

2. Michael CW, Faquin W, Jing X, Kaszuba F, Kazakov J, Moon E, Toloza E, Wu RI and Moreira AL: Committee II: Guidelines for cytologic sampling techniques of lung and mediastinal lymph nodes. Diagn Cytopathol 46: 815-825, 2018.

3. Vilmann P, Clementsen PF, Colella S, Siemsen M, De Leyn P, Dumonceau JM, Herth FJ, Larghi A, Vazquez-Sequeiros E, Hassan C, et al: Combined endobronchial and esophageal endosonography for the diagnosis and staging of lung cancer: European society of gastrointestinal endoscopy (ESGE) guideline, in cooperation with the European respiratory society (ERS) and the European society of thoracic surgeons (ESTS). Endoscopy 47: 545-559, 2015.

4. Xu C, Qin L, Lei W, Jiang J, Ni C and Huang J: The role of endobronchial ultrasound-guided transbronchial needle aspiration liquid-based cytology in the diagnosis of mediastinal lymphadenopathy. Diagn Cytopathol 48: 316-321, 2020.

5. Gupta N, Klein M, Chau K, Vadalia B, Khutti S, Gimenez C and Das K: Adequate at rapid on-site evaluation (ROSE), but inadequate on final cytologic diagnosis: Analysis of 606 cases of endobronchial ultrasound-guided trans bronchial needle aspirations (EBUS-TBNA). Diagn Cytopathol 47: 367-373, 2019.

6. James WE, Koutroumpakis E, Saha B, Nathani A, Saavedra L, Yucel RM and Judson MA: Clinical features of extrapulmonary sarcoidosis without lung involvement. Chest 154: 349-356, 2018.

7. Madan K, Dhungana A, Mohan A, Hadda V, Jain D, Arava S, Pandey RM, Khilnani GC and Guleria R: Conventional transbronchial needle aspiration versus endobronchial ultrasound-guided transbronchial needle aspiration, with or without rapid on-site evaluation, for the diagnosis of sarcoidosis: A randomized controlled trial. J Bronchology Interv Pulmonol 24: 48-58, 2017.

8. Smojver-Jezek S, Peros-Golubicić T, Tekavec-Trkanjec J, Mazuranić I and Alilović M: Transbronchial fine needle aspiration cytology in the diagnosis of mediastinal/hilar sarcoidosis. Cytopathology 18: 3-7, 2007.

9. Odronic SI, Maskovyak AE, Springer BS, Dyhdalo KS, Abdul-Karim FW and Booth CN: Utility and morphologic features of granulomas on rapid on-site evaluation of endobronchial ultrasonography-guided fine-needle aspiration. J Am Soc Cytopathol 3: 79-85, 2014.

10. Gupta D, Dadhwal DS, Agarwal R, Gupta N, Bal A and Aggarwal AN: Endobronchial ultrasound-guided transbronchial needle aspiration vs. conventional transbronchial needle aspiration in the diagnosis of sarcoidosis. Chest 146: 547-556, 2014

11. Sandoh K, Ishida M, Okano K, Miyasaka C, Mori S, Tokuhara M, Suzuki R, Okazaki T, Nakamura N and Tsuta K: Utility of endoscopic ultrasound-guided fine-needle aspiration cytology in rapid on-site evaluation for the diagnosis of gastric submucosal tumors: Retrospective analysis of a single-center experience. Diagn Cytopathol 47: 869-875, 2019.

12. Shield PW, Cosier J, Ellerby G, Gartrell M and Papadimos D: Rapid on-site evaluation of fine needle aspiration specimens by cytology scientists: A review of 3032 specimens. Cytopathology 25: 322-329, 2014. 